\title{
2899. Performance improvement of a sigma-delta modulator using fractional-order disturbance observer
}

\author{
Chi $\mathrm{Xu}^{1}, \mathrm{Yu} \mathrm{Jin}^{2}$, Jingtong $\mathrm{Na}^{3}$ \\ ${ }^{1,2}$ College of Information Science and Technology, Beijing University of Chemical Technology, \\ Beijing, China \\ ${ }^{3}$ College of Electrical and Information engineering, Dalian Jiaotong University, Dalian, China \\ ${ }^{2}$ Corresponding author \\ E-mail: 12016400134@mail.buct.edu.cn, ${ }^{2}$ jiny@mail.buct.edu.cn, ${ }^{3} 13624099110 @ 163 . c o m$
}

Received 8 August 2017; received in revised form 4 December 2017; accepted 14 December 2017 DOI https://doi.org/10.21595/jve.2017.18954

Check for updates

Copyright (C) 2018 Chi Xu, et al. This is an open access article distributed under the Creative Commons Attribution License, which permits unrestricted use, distribution, and reproduction in any medium, provided the original work is properly cited.

\begin{abstract}
This paper proposes a novel design of a high order closed-loop Sigma-Delta modulator using a fractional-order disturbance observer, which can reduce the influence of possible dispersions of the sensing element and offset the effect of the external disturbance. The proof mass can maintain near its position of equilibrium by using the proposed fractional-order disturbance observer, which ensures a better control of the proof mass displacement, and the small proof mass displacement can enhance the sensor resolution and the overall system performance. Fractional-order disturbance observer possesses stronger robust stability of interference elimination, because the introduction of fractional calculus makes Q-filter expanding from an integer domain to a real-number domain. Experimental results from the proposed architecture shows an increase of more than $2 \mathrm{X}$ improved in signal quantization noise ratio compared to the pure high-order closed-loop Sigma-Delta modulator, and also the proposed Sigma-Delta modulator with a fractional-order disturbance observer improves the performance of disturbance attenuation and system robustness.
\end{abstract}

Keywords: fractional-order disturbance observer, Sigma-Delta modulator, Q-filter, robust stability.

\section{Introduction}

The fast development of micro-processing and integrated circuit technology were the main driver of the widely application for the MEMS (Micro-Electromechanical System) accelerometer $[1,2]$. MEMS accelerometers are commonly used in automotive applications, biomedical domain and navigation system [3-5] thanks to their small size, low cost, and low power consumption, etc. MEMS accelerometers using a capacitive sensing element incorporated in Sigma-Delta $(\Delta \Sigma)$ modulator closed-loop control systems with electrostatic feedback. MEMS accelerometer systems consist of chips developed separately may not work properly together when they were integrated together even each chip might be designed and optimized in its own develop project. MEMS accelerometer requires system level integrated design and development. As we all know that an advanced MEMS accelerometer system contains several features such as (1) feedback system with MEMS sensor in the loop; (2) system with sensing and digitization in one step; (3) system with multi-physics principles; (4) $\Sigma$ - $\Delta$ closed-loop system with force rebalance.

Among various techniques for implementing the MEMS accelerometer, $\Delta \Sigma$ modulator provides the combined benefits of force feedback and inherent analog-to-digital conversion [6]. The sensing element of MEMS accelerometer can be modeled as a mass-spring-damper system, which provides a 2 nd order dynamics. Therefore, a traditional MEMS accelerometer is mainly focused on using the sensing element as a low-pass filter to form a 2 nd order $\Delta \Sigma$ modulator [7]. At present, many researchers have mainly focused on using the additional electronic integrator as a loop integrator to form the high order closed-loop $\Delta \Sigma$ modulator to improve its SNR and noise performance [8]. There are a lot of advantages to the high order closed-loop $\Delta \Sigma$ modulator, nevertheless such this architecture may suffer from the overall system instability. As discussed, a 
$\Delta \Sigma$ modulator, which can both achieve high SNR, outstanding noise performance and also ensure the system stable, is much difficulty to be designed. It is shown that the Sigma-Delta modulator system can be studied as a nonlinear dynamical system with feedback control [9]. Representing a modulator as "plant" and "controller" transforms a modulator design problem into a control design problem. Therefore, many researchers introduced several advanced control techniques to Sigma-Delta modulator. In ref $[10,11]$, a high-order Sigma-Delta modulator design by slide mode control is proposed, which can precisely predict the modulator performs and reduce the nonlinear effect from the 1-bit comparator; In ref [12], a Kalman state estimator is applied to a Sigma-Delta micro-accelerometer which can enhance the resolution of the Sigma-Delta modulator. In this paper, we present an approach that ensures a better control of the proof mass displacement thanks to the proposed fractional-order disturbance observer, which is able to improve the noise performance and system robust stability.

In practice, a physical motion system cannot be exactly the same as any mathematical model, no matter how the model is obtained [13]. In the system of a MEMS accelerometer, a precise mathematic model of the sensing element which may not be readily available because of the external disturbance (temperature, pressure) or dispersions in the manufacturing process. Thus, the mismatch between the real and ideal parameters can result in relatively large proof mass displacements and as a consequence in a decrease of the overall system performance. The concept of disturbance observer (DOB) was proposed in ref [14], which is able to improve the ability of disturbance rejection. The filter in DOB belongs to the low-pass filter called Q-filter. The so-called Q-filter has three adjustable parameters, including the order, the relative degree and the bandwidth. Therefore, compared to integral action, DOB offers more opportunities to improve the performance of the control system (rapidity, stability, phase shift etc.) through tuning the three parameters of Q-filter. In ref. [15], a fractional-order disturbance observer (FO-DOB) based on a fractional-order Q-filter was proposed and thus extending the integer-order disturbance observer to a new fractional-order one. The fractional-order Q-filter is employed to make a tradeoff between the robust stability and the disturbance suppressing force. Therefore, in this paper, FO-DOB is employed to observe and compensate the disturbances and model mismatch of the sensing element, which is able to make the overall system have better robust stability against variation of mathematic model of the sensing element and then ensure MEMS accelerometer achieve high SNR and satisfactory noise performance.

The remainder part of this paper is organized as follows: in Section 2, a basic architecture of FO-DOB and parameters design in FO-DOB are introduced; in Section 3, the proposed design of a high order closed-loop $\Delta \Sigma$ modulator with a FO-DOB is presented. Then simulation results of the proposed design architecture are demonstrated and analyzed in Section 4. Finally, conclusions are given in Section 5 .

\section{Continuous-time fractional-order disturbance observer}

The basic idea of the conventional DOB is to apply a nominal inverse model of the plant to observe the disturbance which caused by external interference and parameters variation, and then an equivalent compensation is generated by DOB. The basic architecture of DOB is depicted in Fig. 1.

In Fig. $1, G_{p}(s)$ is the transfer function of the plant model, $d$ is the equivalent disturbance, $\tilde{d}_{f}$ is the observing value of $d, c$ and $y$ are the input and output of DOB, respectively. By observation from Fig. 1, the observing value $\tilde{d}_{f}$ can be written as:

$\tilde{d}_{f}=(u+d) \cdot G_{p}(s) \cdot G_{p}^{-1}(s)-u=d$.

Unfortunately, the implementation of the conventional DOB encounters three key problems in a practical system [8] as follows: 
1) In general cases, the relative order of $G_{p}(s)$ is not equal to zero, therefore the inverse of the plant model cannot be obtained.

2) The transfer function of the plant model cannot describe the practical system precisely, that is to say $G_{p}(s)$ is not totally accurate.

3) The existence of the measurement noise will deteriorate the control performance of control system.

In order to solve the above three problems, a Q-filter and a transfer function $G_{n}(s)$ are introduced, which is shown as in Fig. 2.

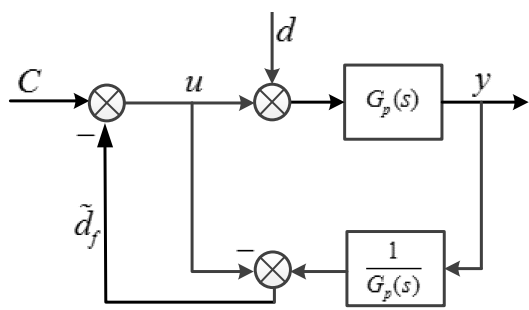

Fig. 1. Basic architecture of DOB

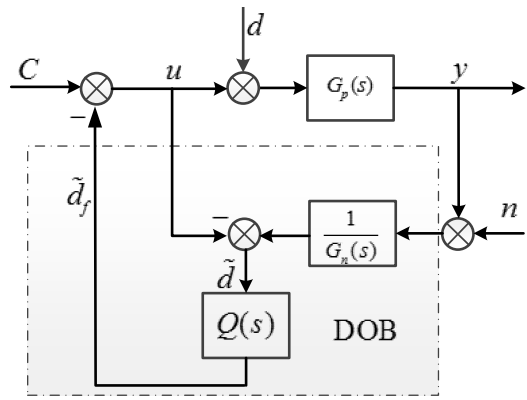

Fig. 2. Block diagram of DOB

In Fig. 2, $Q(s)$ is a low-pass filter, which is used to restrict the effective bandwidth of DOB. $\tilde{d}$ and $\tilde{d}_{f}$ are the disturbance observe before and after by the Q-filter.

From the conventional DOB shown in Fig. 2, the signal transfer function $G_{c y}(s)$, the disturbance transfer function $G_{d y}(s)$ and the noise transfer function $G_{n y}(s)$ can be written as:

$$
\begin{aligned}
& G_{c y}(s)=\frac{Y(s)}{C(s)}=\frac{G_{p}(s) G_{n}(s)}{G_{n}(s)+Q(s)\left(G_{p}(s)-G_{n}(s)\right)} \\
& G_{d y}(s)=\frac{Y(s)}{D(s)}=\frac{G_{p}(s) G_{n}(s)(1-Q(s))}{G_{n}(s)+Q(s)\left(G_{p}(s)-G_{n}(s)\right)}, \\
& G_{n y}(s)=\frac{Y(s)}{N(s)}=\frac{G_{p}(s) Q(s)}{G_{n}(s)+Q(s)\left(G_{p}(s)-G_{n}(s)\right)},
\end{aligned}
$$

where, we assume that the cutoff frequency of the low-pass Q-filter is $\omega_{q}$. When $\omega<\omega_{q}$, $Q(s) \approx 1$, taking $Q(s) \approx 1$ into Eqs. (2), (3) and (4), the signal transfer function $G_{c y}(s) \approx G_{n}(s)$, the disturbance transfer function $G_{d y}(s) \approx 0$ and the noise transfer function $G_{n y}(s) \approx 1$; and when $\omega>\omega_{q}, Q(s) \approx 0$, taking $Q(s) \approx 0$ into Eqs. (2), (3) and (4), the signal transfer function $G_{c y}(s) \approx G_{p}(s)$, the disturbance transfer function $G_{d y}(s) \approx G_{p}(s)$ and the noise transfer function $G_{n y}(s) \approx 0$. By observation from $G_{c y}(s), G_{d y}(s)$ and $G_{n y}(s)$, we can find that the external disturbance can be suppressed by designing the low-pass Q-filter. In other words, the design of Q-filter is a key part in DOB design. In particular, the relative order of the low-pass Q-filter should 
be more than that of $G_{n}(s)$ to maintain $Q(s) G_{n}^{-1}(s)$ being a regular rational transfer function, and the bandwidth design of Q-filter should make a tradeoff between robust stability and disturbance elimination force of the overall system.

The relation between the real model $G_{p}(s)$ and ideal model $G_{n}(s)$ can be expressed by:

$G_{p}(s)=G_{n}(s)(1+\Delta(s))$

where $\Delta(s)$ is a uncertain transfer function, which contains variable parameters of the plant model. The robust stability criterion of the uncertain system is discussed in [16], the sufficient criterion of robust stability for Q-filter is expressed as follows:

$\|\Delta(s) Q(s)\| \leq 1$

In order to simplify design process of the low-pass Q-filter, an equivalent block diagram of DOB is shown in Fig. 3.

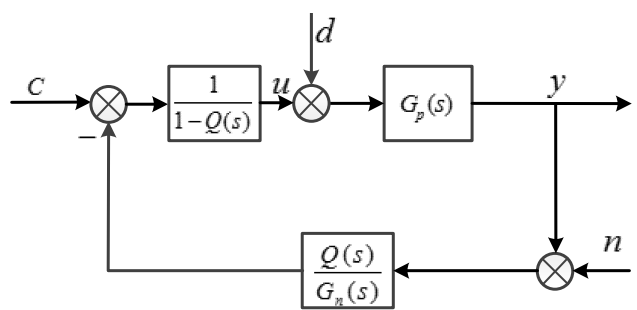

Fig. 3. Equivalent block diagram of DOB

It can be seen from the block $1 /(1-Q(s))$ shown in Fig. 3, the disturbance observer is a high gain technique when $Q(s) \rightarrow 1$. To simplify the analysis of uncertain transfer function $\Delta(s)$, here we assume $e^{-s T_{d}}$ is the only source of uncertain dynamics, so $G_{p}(s)$ can be rewritten as:

$G_{p}(s)=G_{n}(s) e^{-s T_{d}}$

By observation from Eqs. (5) and (7), the uncertain transfer function $\Delta(s)$ is written as:

$\Delta(s)=e^{-s T_{d}}-1$.

In this paper, the low-pass filter $Q(s)$ can be expressed as:

$Q(s)=\frac{1}{(\tau s+1)^{n}}$

where cutoff frequency $\omega_{q}=1 / \tau$, here we set $T_{d}=0.2 \mathrm{~ms}, n=1, \omega_{q 1}=50 \mathrm{rad} / \mathrm{sec}$, $\omega_{q 2}=100 \mathrm{rad} / \mathrm{sec}, \omega_{q 3}=500 \mathrm{rad} / \mathrm{sec}$, so the uncertain transfer function $\Delta(s)$ and three $Q(s)$ are rewritten as follows:

$\Delta(s)=e^{-0.0002 \cdot s}-1$,

$Q_{2}(s)=\frac{1}{0.01 s+1}$

$Q_{1}(s)=\frac{1}{0.002 s+1}$,

$Q_{3}(s)=\frac{1}{0.02 s+1}$. 
The robust stability of DOB with respect to the cutoff frequency $\omega_{q}$ of $Q(s)$ is depicted in Fig. 4.

It is obvious from Fig. 4 that the robust stability criterion Eq. (6) is violated when $\omega_{q}=500 \mathrm{rad} / \mathrm{sec}$. The magnitude responses of $Q_{2}(s)$ and $Q_{3}(s)$ are below the curve of $1 / \Delta$, so the robust stability criterion can be satisfied when $\omega_{q} \leq 100 \mathrm{rad} / \mathrm{sec}$. Also, here we set $T_{d}=0.2 \mathrm{~ms}, \omega_{q}=100 \mathrm{rad} / \mathrm{sec}$ and $n=1,2,3$ to inspect the variations of the robust stability of DOB with respect to relative order $n$ of the $Q(s)$. Fig. 5 shows three curves with different relative order $n=1,2,3$.

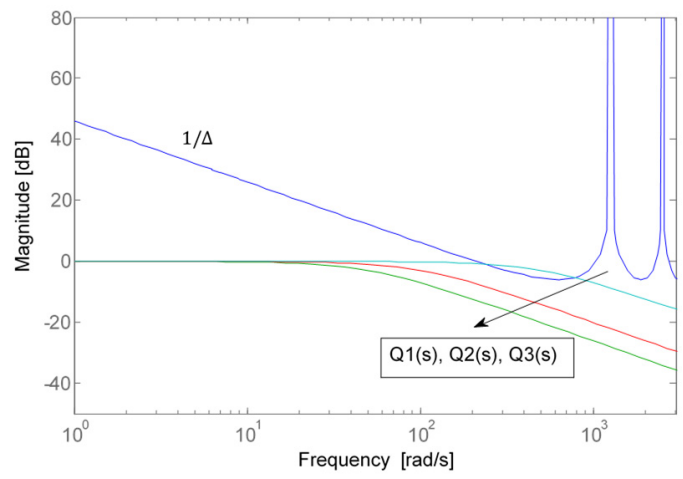

Fig. 4. The robust stability of DOB with different cutoff frequency

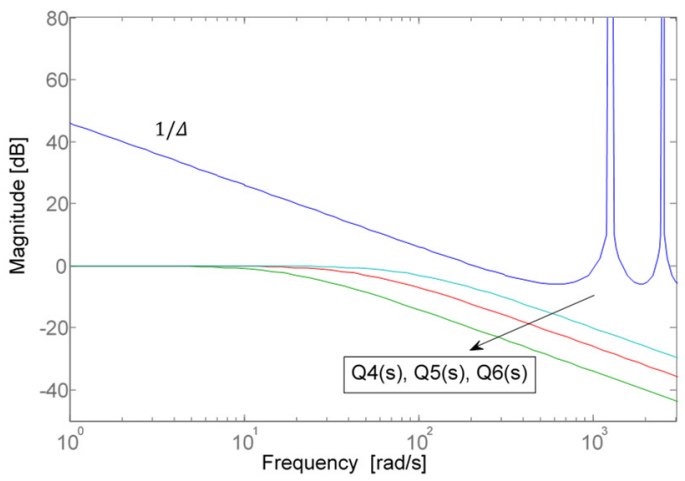

Fig. 5. The robust stability of DOB with different orders

By observation from Fig. 5, the higher relative order of $Q(s)$, the better robust stability of DOB. In ref. [14], the cutoff frequency $\omega_{q}$ is always determined according to the disturbance attenuation requirement in advance. Therefore, the relative order of the low-pass $Q(s)$ is the only knob to design. As discussed, if the relative order can be tuned in the real-number domain instead of the integer domain, the tuning range will be broader dramatically, while the relative order is selected in real-number domain, as a consequence, a traditional disturbance observer belongs to a fractional-order disturbance observer. Therefore, FO-DOB possesses more flexibility than the conventional DOB for disturbance elimination in that the relative order of the low-pass Q-filter can be tuned in a real-number domain. In the field of FO-DOB, the low-pass $Q(s)$ has become fractional-order low-pass filter $Q_{\alpha}(s)$ and the fractional order $\alpha$ can be continuously tuned to improve the dynamic performance of FO-DOB. In this paper, the fractional-order low-pass filter $Q_{\alpha}(s)$ is expressed as:

$Q_{\alpha}(s)=\frac{1}{(\tau s+1)^{\alpha}}$ 
where $\alpha \in R, \tau=1 / \omega_{q}$, in this paper, a continuous integer-order filter $Q_{I}(s)$ is used to approximate the fractional-order filter $Q_{\alpha}(s)$ in a selected frequency bandwidth $\left[\omega_{b}, \omega_{h}\right]$. General form of the integer-order filter $Q_{I}(s)$ is given as follows:

$Q_{I}(s)=K \prod_{k=-N}^{N} \frac{s+\omega_{k}}{s+\omega_{k}^{\prime}}$

The steps of this approximation method are present in [17]. The fractional-order filter $Q_{\alpha}(s)$ is approximated by a fifth order integer-order filter $Q_{I}(s)$ in bandwidth range $[100,1000]$, which is expressed as:

$Q_{\alpha}(s) \approx Q_{I}(s)=\frac{b_{5} s^{5}+b_{4} s^{4}+b_{3} s^{3}+b_{2} s^{2}+b_{1} s+b_{0}}{a_{5} s^{5}+a_{4} s^{4}+a_{3} s^{3}+a_{2} s^{2}+a_{1} s+a_{0}}$.

Fig. 6 shows the bode curves of the fractional-order filter $Q_{\alpha}(s)$ with different $\alpha$.

Here we set $\tau=0.01 \mathrm{~s}, \alpha=1.6$, so the fractional-order low-pass filter $Q_{\alpha}(s)$ is written as:

$Q_{1.6}(s)=\frac{\left(\begin{array}{c}s^{5}+2.168 \times 10^{4} \cdot s^{4}+1.168 \times 10^{8} \cdot s^{3} \\ +2.754 \times 10^{11} \cdot s^{2}+1.977 \times 10^{14} \cdot s+3.981 \times 10^{16}\end{array}\right)}{\left(\begin{array}{c}1585 \cdot s^{5}+7.783 \times 10^{6} \cdot s^{4}+1.096 \times 10^{10} \cdot s^{3} \\ +5.248 \times 10^{12} \cdot s^{2}+8.632 \times 10^{14} \cdot s+3.981 \times 10^{16}\end{array}\right)}$

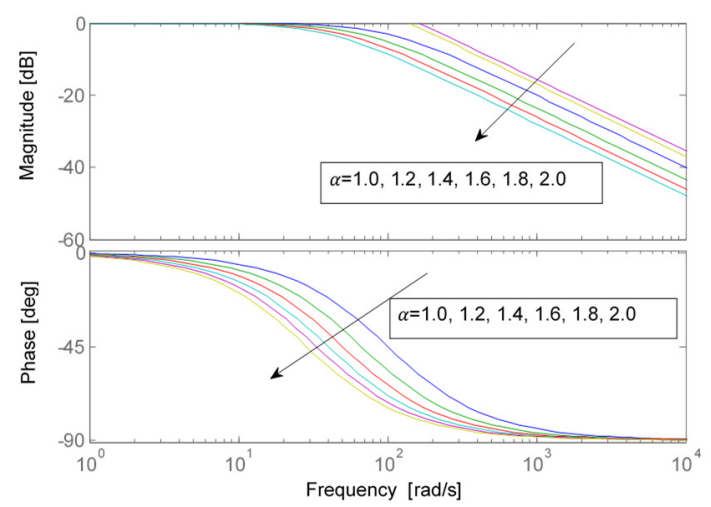

Fig. 6. Bode curves of $Q_{\alpha}(s)$ with different $\alpha$

\section{Implementation in the case of a $\Delta \Sigma$ modulator}

The general system block diagram of $\Delta \Sigma$ modulator is shown in Fig. 7 .

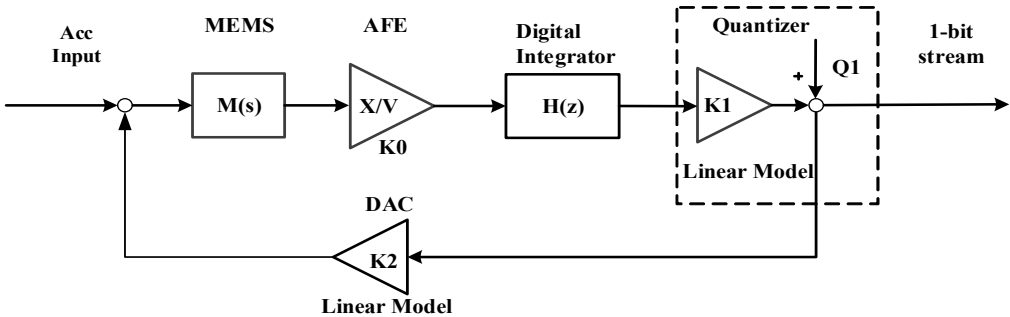

Fig. 7. Block diagram of $\Delta \Sigma$ modulator system

The loop is consisted of an electromechanical sensing element, a charge amplifier, a digital integrator, and a 1-bit quantizer. In Fig. $1, K_{0}$ is the gain of AFE, $H(\mathrm{z})$ is a digital integrator, $K_{1}$ 
is the quantizer gain, $Q_{1}$ is the quantization noise of 1-bit quantizer, and $K_{2}$ is the equivalent linear model of 1-bit DAC feedback. Unfortunately, the relationship of 1-bit quantizer input and output is nonlinear, here, a quasi-linear model of the 1-bit quantizer is presented in Fig. 1, where the quantizer output is equal to the sum of quantization noise $Q_{1}$ and quantizer input with a quantization gain $k_{1}$.

The sensing element can be modeled as a mass-spring-damper system, a 2nd order dynamics with transfer function $M(s)$ that can be expressed as Eq. (18):

$M(s)=\frac{1}{s^{2}+\frac{b}{m} s+\frac{k}{m}}$,

where $m$ is the proof mass, $b$ is the damping coefficient and $k$ is the spring constant. Due to the parametric yield errors, $b$ and $k$ are not always the actual values caused by manufacturing process. So, FO-DOB is employed to observe and compensate the model mismatch of the proof mass. The proposed architecture of the closed-loop $\Delta \Sigma$ modulator with a FO-DOB is given in Fig. 8.

In Fig. $8, M^{-1}(s)$ is the inverse model of the sensing element $M(s), Q_{\alpha}(s)$ is the fractional-order Q-filter, $e^{-s \cdot T_{d}}$ is the time delay. By observation from Fig. 8, FO-DOB is employed to observe and compensate the disturbances and model mismatch of the sensing element. The effects of the employed FO-DOB are shown in the following.

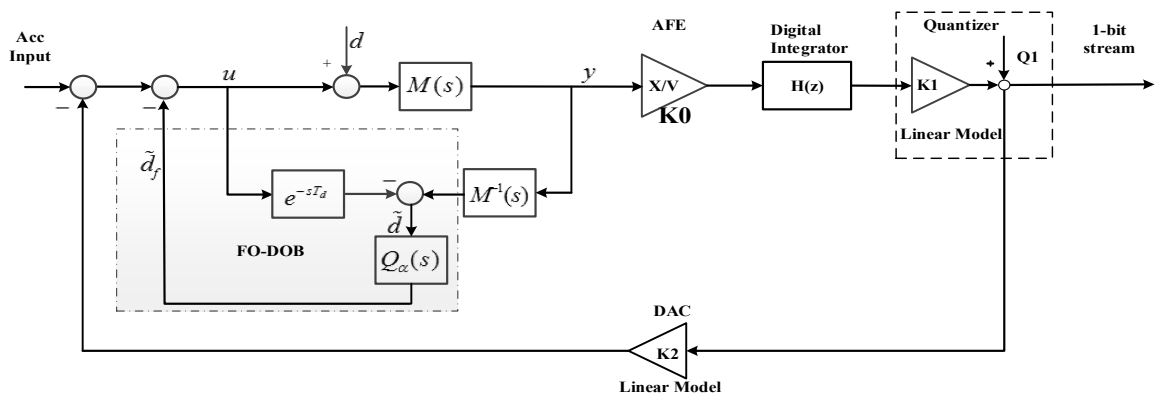

Fig. 8. Block diagram of the proposed $\Delta \Sigma$ modulator system

\section{Simulation}

Firstly, the Simulink model of the proposed $\Delta \Sigma$ modulator with a FO-DOB is developed shown in Fig. 9.

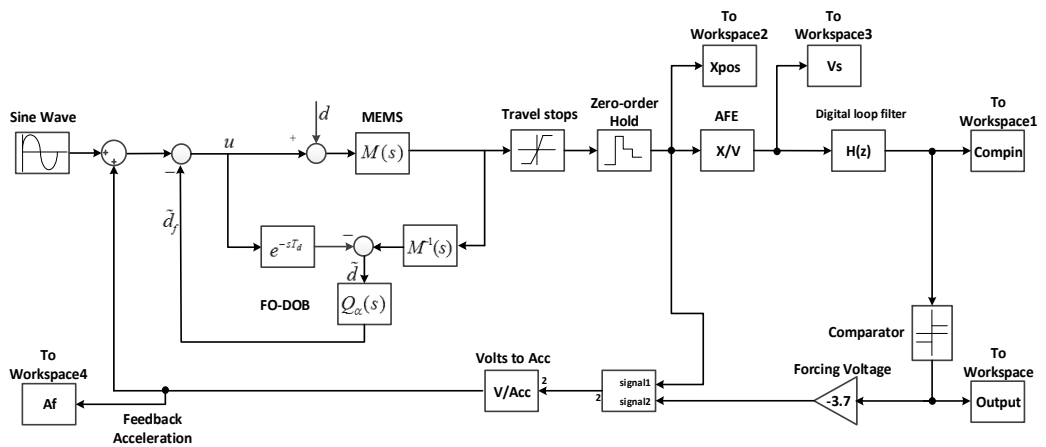

Fig. 9. Simulink model of the proposed $\Delta \Sigma$ modulator with a FO-DOB

In Fig. 9, the oversampling ratio (OSR) needs to be specified in the $\Delta \Sigma$ modulator system. Here, we choose sample frequency as $128 \mathrm{kHz}$, and OSR $=64$. The mechanical and system parameters 
in the proposed architecture of the closed-loop $\Delta \Sigma$ modulator with a FO-DOB are listed in Table 1.

Table 1. Parameters of the proposed architecture

\begin{tabular}{|c|c|}
\hline Parameter & Value \\
\hline Proof mass $m[\mathrm{mg}]$ & 20 \\
\hline Damping coefficient $b[\mathrm{Ns} / \mathrm{m}]$ & $2.4 \times 10^{-3}$ \\
\hline Spring constant $k[\mathrm{~N} / \mathrm{m}]$ & 100 \\
\hline AFE gain $k_{0}[\mathrm{~V} / \mathrm{m}]$ & $4.55 \times 10^{7}$ \\
\hline Comparator gain $k_{1}$ & 1 \\
\hline Feedback gain $k_{2}\left[\mathrm{~m} / \mathrm{s}^{2}\right]$ & 49.05 \\
\hline External disturbance & $3.4 \mathrm{ng} / \sqrt{\mathrm{Hz}}$ \\
\hline
\end{tabular}

Two numerical experiments of the proposed architecture have been performed in the following. Case one. A 5 th order closed-loop $\Delta \Sigma$ modulator with the FO-DOB.

In this case, we choose the digital integrator $H(z)$ for the closed-loop $\Delta \Sigma$ modulator as follows:

$H(z)=\frac{1000 z^{3}-2907 z^{2}+2844 z-935.9}{z^{3}-2.517 z^{2}+2.034 z-0.5168}$.

Due to the system of sensing element responds quickly, $T_{d}$ in Fig. 8 is set as $0.2 \mathrm{~ms}$, and $\alpha$ has been adjusted empirically, so finally $\alpha$ is selected as 2.4 to inspect the effects of FO-DOB.

Taking $\alpha=2.4$ in Eq. (14):

$Q_{2.4}(s)=\frac{1}{(0.0002 s+1)^{2.4}}=\frac{1}{(0.0002 s+1)^{2} \cdot(0.0002 s+1)^{0.4}}$.

From Eq. (20), $Q_{2.4}(s) \cdot M^{-1}(s)$ can be obtained. Taking $H(\mathrm{z})$, the linearized parameters $k_{0}$, $k_{1}, k_{2}$ and the major transfer function $M(s)$ into Fig. 9, The corresponding SNR is $124.454 \mathrm{~dB}$ which is $2 \mathrm{X}$ improved comparing to 5 th order $\Delta \Sigma$ modulator only. The PSD plot of the proposed $\Delta \Sigma$ modulator is shown in Fig. 10. Comparing to the only 5 th order $\Delta \Sigma$ modulator, the proposed $\Delta \Sigma$ modulator with the FO-DOB not only performs better SNR, but also lower noise floor.

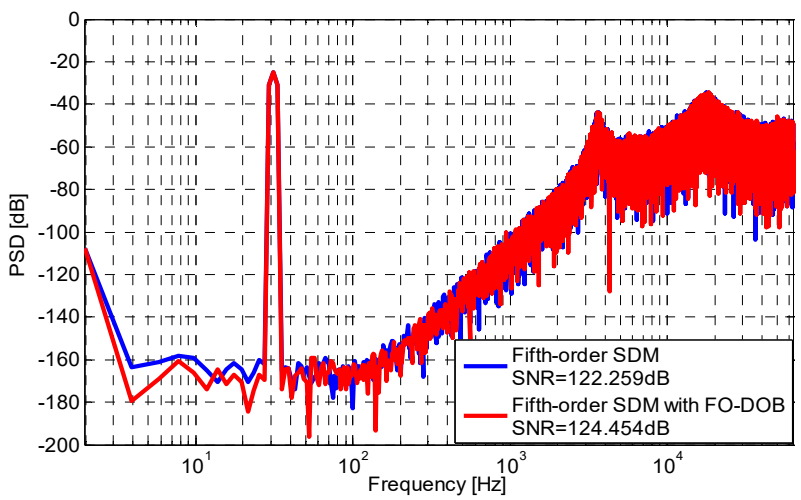

Fig. 10. PSD of the 5 th order $\Delta \Sigma$ modulator using FO-DOB

Case two. A 6th order closed-loop $\Delta \Sigma$ modulator with FO-DOB.

In this case, we choose the digital integrator $H(z)$ for the closed-loop $\Delta \Sigma$ modulator as follows:

$H(z)=\frac{0.001 \times\left(1.635 z^{4}+0.381 z^{3}-0.9485 z^{2}-0.4088 z+0.08612\right)}{z^{4}+0.5 z^{3}-0.75 z^{2}-0.25 z+0.0625}$.

Taking $H(\mathrm{z})$, the linearized parameters $k_{0}, k_{1}, k_{2}$ and the major transfer function $M(s)$ into 
Fig. 9, The corresponding SNR is $144.265 \mathrm{~dB}$ which is $2 \mathrm{X}$ improved comparing to 6th order $\Delta \Sigma$ modulator only. The PSD plot of the proposed $\Delta \Sigma$ modulator with FO-DOB is shown in Fig. 10. Comparing to the only 6th order $\Delta \Sigma$ modulator, the proposed $\Delta \Sigma$ modulator not only performs better SNR, but also lower noise floor.

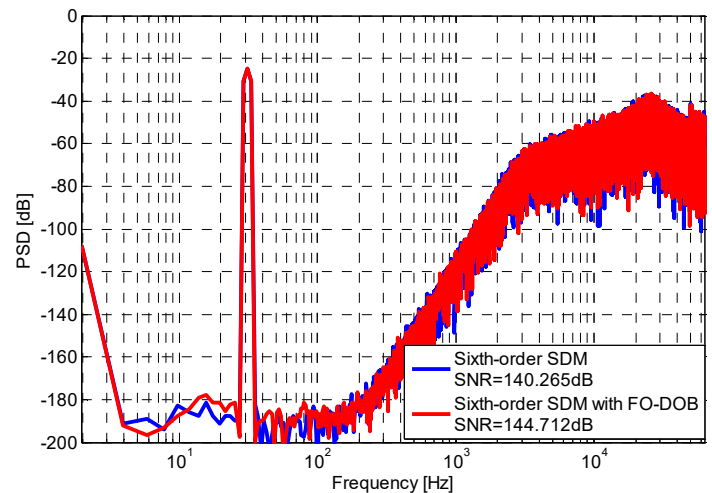

Fig. 11. PSD of the 6 th order $\Delta \Sigma$ modulator using FO-DOB

Further simulation are also observed at system response with different spring constant $\mathrm{k}$ and damping coefficient $\mathrm{b}$, For instance, taking different spring constants $k_{s 1}=60 \mathrm{~N} / \mathrm{m}$, $k_{s 2}=120 \mathrm{~N} / \mathrm{m}, k_{s 3}=240 \mathrm{~N} / \mathrm{m}$ in MEMS transfer function to verify the robustness of the proposed $\Delta \Sigma$ modulator with the FO-DOB, Fig. 12 and Fig. 13 show the PSD plot of the proposed 5 th order and 6th order $\Delta \Sigma$ modulator with different spring constant $k_{s 1}, k_{s 2}, k_{s 3}$ using FO-DOB.

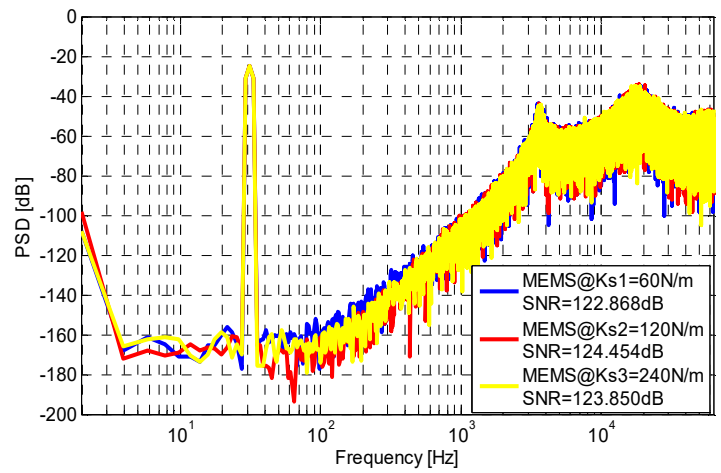

Fig. 12. PSD of the 5 th order $\Delta \Sigma$ modulator with different spring constant $k$

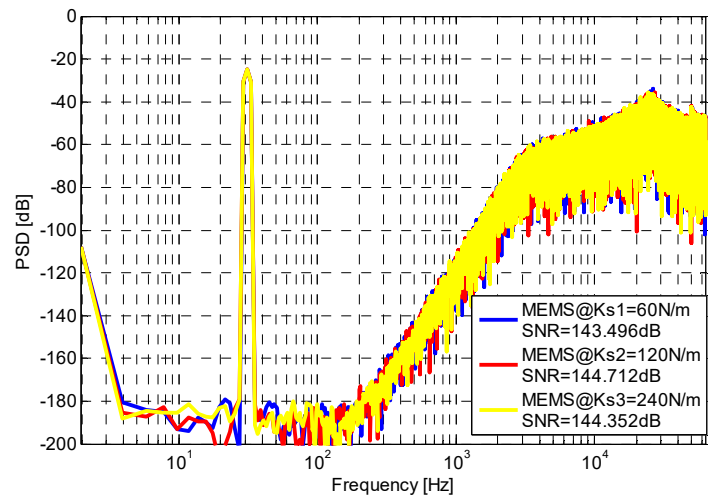

Fig. 13. PSD of the 6th order $\Delta \Sigma$ modulator with different spring constant $k$ 
Also, taking $b_{1}=1.8 \times 10^{-3} \mathrm{Ns} / \mathrm{m}, b_{2}=3.6 \times 10^{-3} \mathrm{Ns} / \mathrm{m}, b_{3}=5.4 \times 10^{-3} \mathrm{Ns} / \mathrm{m}$ in MEMS transfer function, Fig. 13 and Fig. 14 show the PSD plot of the proposed 5th order and 6th order $\Delta \Sigma$ modulator with different damping coefficient $b$.

Simulation results show that SNR and the noise floor of the three sensing elements with different $k$ and three sensing elements with different $b$ only present an acceptable slight fluctuation which indicate that the proposed $\Delta \Sigma$ modulator using FO-DOB has strong robustness against to the sensitivity of MEMS devices.

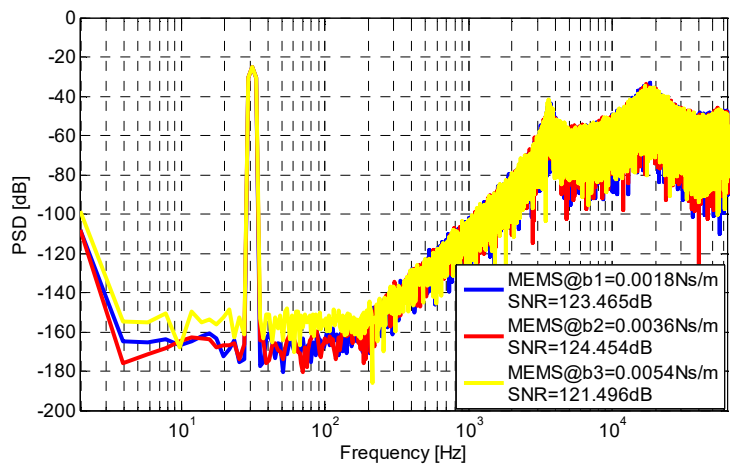

Fig. 14. PSD of the 5 th order $\Delta \Sigma$ modulator with different damping coefficient $b$

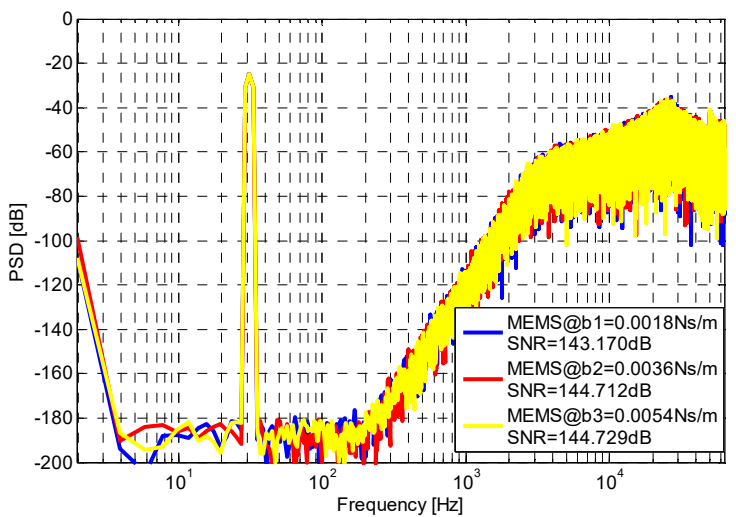

Fig. 15. PSD of the 6th order $\Delta \Sigma$ modulator with different damping coefficient $b$

\section{Conclusions}

A novel design of a high order closed-loop Sigma-Delta modulator using a fractional-order disturbance observer is present in this paper. The proposed 5 th order Sigma-Delta modulator using FO-DOB achieved SNR $=124.454 \mathrm{~dB}$ in simulation and noise floor under $-170 \mathrm{~dB}$ in frequency of $[5-150 \mathrm{~Hz}]$ and the proposed 6th order Sigma-Delta modulator using FO-DOB achieved $\mathrm{SNR}=144.712 \mathrm{~dB}$ in simulation and noise floor under $-190 \mathrm{~dB}$ in frequency of [5-150 Hz]. The numerical experiments also show the improved robust stability of the proposed Sigma-Delta modulator using fractional-order disturbance observer comparing to the pure 5th order and 6th order Sigma-Delta modulator. This study can promote the development of high performance of MEMS accelerometer, also provide a scientific and technical supports for the application of fractional-order disturbance observer.

\section{Acknowledgements}

This work was supported by the research fund to the top scientific and technological innovation 
team from Beijing University of Chemical Technology (No. buctylkjcx06).

\section{References}

[1] Marek J. MEMS for automotive and consumer electronics. IEEE International on Solid-State Circuits Conference Digest of Technical Papers, 2010.

[2] Aysu A., Ghalaty N. F., Franklin Z., Yali M. P., Schaumont P. Digital fingerprints for low-cost platforms using MEMS sensors. Proceedings of the Workshop on Embedded Systems Security, New York, 2013.

[3] Lin H., Zhang Y., Long F., Mei F., Yu M., Lin F., Yao L., Jiang X. Digital noise-coupling technique for delta-sigma modulators with segmented quantization. IEEE Transactions on Circuits and Systems II: Express Briefs, Vol. 61, Issue 6, 2014, p. 403-407.

[4] Bafandeh A., Yavari M. Digital calibration of amplifier finite DC gain and gain bandwidth in mash $\Sigma \Delta$ modulators. IEEE Transactions on Circuits and Systems II: Express Briefs, Vol. 63, Issue 4, 2016, p. 321-325.

[5] Luo X. C., Feng J. A monolithic MEMS gyroscope interface circuit in $0.35 \mu \mathrm{m}$ CMOS. Tien Tzu Hsueh Pao/Acta Electronica Sinica, Vol. 42, Issue 9, 2014, p. 1868-1872.

[6] Keller M., Buhmann A., Ortmanns M., Manoli Y. A method for the discrete-time simulation of continuous-time sigma-delta modulators. IEEE International Symposium on Circuits and Systems, 2007, p. 241-244.

[7] Saxena G. D., Thamarai V. Modeling and simulation of high performance sixth order sigma-delta MEMS accelerometer. International Conference on Computational Intelligence and Communication Networks, 527, p. 531-2011.

[8] Petkov V. P., Boser B. High-order electromechanical modulation in micro-machined inertial sensors. IEEE Transactions Circuits and Systems, Vol. 53, Issue 5, 2006, p. 1016-1022.

[9] Handtmann M., Aigner R., Meckes A., Wachutka G. Sensitivity enhancement of mems inertial sensors using negative springs and active control. Sensors and Actuators A, Vol. 97-98, 2002, p. $153-160$.

[10] Plekhanov S., Shkolnikov I. A., Shtessel Y. B. High order sigma-delta modulator design via sliding mode control. American Control Conference, 2003, p. 897-902.

[11] Yu S. H. Analysis and design of sigma-delta modulators using the theory of sliding modes. International Conference on Signals and Electronic System, Poznan, Poland, 2004, p. 91-94.

[12] Colmet E., Juillard J., Guessab S. Resolution enhancement of a sigma-delta micro-accelerometer using signal prediction. International Conference on MEMS, NANO and Smart Systems, Banff, Canada, 2004, p. 72-79.

[13] Chen Y. Q., Vinagre B. M., Podlubny I. Fractional order disturbance observer for robust vibration suppression. Nonlinear Dynamics, Vol. 38, 2004, p. 355-367.

[14] Kempf C. J., Kobayashi S. Disturbance observer and feedforward design for a high-speed directdrive positioning table. IEEE Transactions on Control System and Technology, Vol. 7, Issue 5, 1999, p. 513-526.

[15] Brahima N. I., Darouach Voos M. H., Michel Z. Design of unknown input fractional-order observers for fractional-order systems. International Journal of Applied Mathematics and Computer Science, Vol. 23, Issue 3, 2013, p. 491-500.

[16] Liu J. K. Advance PID Control and MATLAB Simulation. Publishing House of Electronics Industry, Beijing, China, 2003.

[17] Chen H., Chen Y. Fractional-order generalized principle of self-support in control system design. Journal of Automatica Sinica, Vol. 3, Issue 4, 2016, p. 430-441.

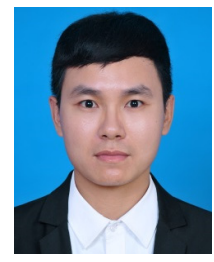

Chi Xu is with the College of Information Science and Technology, Beijing University of Chemical Technology, Beijing China. His current research interests include Sigma-Delta modulator, fractional-order control theory, intelligent optimization algorithm. 

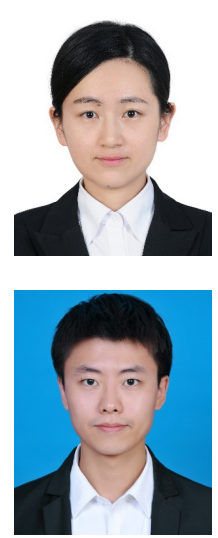

Yu Jin received Ph.D. degree in Graduate School of Information, Production and Systems from Waseda University, Japan, in 2014. Now she works at the College of Information Science and Technology of Beijing University of Chemical Technology, Beijing China. Her current research interests include LSI design and fabrication, high performance ADC design.

Jingtong $\mathbf{N a}$ is with the College of Electrical and Information Engineering, Dalian Jiaotong University, Dalian, China. His current research interests include fractional-order control theory, intelligent control. 